\title{
Toxicidade de óleos essenciais de Piper aduncum e Piper hispidinervum em Sitophilus zeamais
}

\author{
Joelma Lima Vidal Estrela( ${ }^{(1)}$, Murilo Fazolin( ${ }^{(1)}$, Valdomiro Catani( ${ }^{(1)}$, Marcio Rodrigues Alécio ${ }^{(1)}$ \\ e Marilene Santos de Lima ${ }^{(1)}$
}

\begin{abstract}
(1)Embrapa Acre, Caixa Postal 321, CEP 69908970 Rio Branco, AC. E mail: joelma@cpafac.embrapa.br, murilo@cpafac.embrapa.br, catani@cpafac.embrapa.br

Resumo - Óleos essenciais de Piper aduncum L. e Piper hispidinervum C.DC. (Piperaceae) foram avaliados quanto ao efeito inseticida em Sitophilus zeamais Motsch. por ação de contato, fumigação e tópica. Na determinação da $\mathrm{DL}_{50}$ e $\mathrm{CL}_{50}$, foi utilizada a análise de Probit. Médias porcentuais de mortalidade dos insetos foram comparadas por meio da análise de regressão linear e superposição das barras do erro padrão. S. zeamais foi mais suscetível ao efeito de contato do óleo de $P$. hispidinervum em relação ao de $P$. aduncum, obtendo-se $\mathrm{CL}_{50}$ de 0,51 e $2,87 \mathrm{~mL} \mathrm{~cm}^{-2}$ de óleo, respectivamente. Mortalidade próxima a $100 \%$ foi obtida nas concentrações de 20 e 30\% do óleo de P. hispidinervum. Quanto ao efeito fumigante, a susceptibilidade foi maior no óleo de $P$. aduncum do que no de $P$. hispidinervum. Houve diferença significativa entre os óleos somente nas concentrações de 0,1 e 1,0. A DL ${ }_{50}$ foi semelhante nos dois óleos essenciais por aplicação tópica. No entanto, a mortalidade foi maior com $P$. aduncum. Óleos essenciais de $P$. aduncum e $P$. hispidinervum possuem efeito inseticida em S. zeamais, mas as respostas dependem da concentração e do método de exposição a que o inseto seja submetido.
\end{abstract}

Termos para indexação: Piperaceae, inseticidas botânicos, dilapiol, safrol, inseticidas naturais.

\section{Toxicity of essential oils of Piper aduncum and Piper hispidinervum against Sitophilus zeamais}

\begin{abstract}
Essential oils of Piper aduncum L. and Piper hispidinervum C. DC. (Piperaceae) were tested against Sitophilus zeamais Motsch. Contact, fumigant toxicity and topical effect were tested. Probit analysis was employed in evaluating the $\mathrm{LC}_{50}$ and $\mathrm{LD}_{50}$ response. Linear regression analysis and superposition of the bars (means error standard) were used for comparison of means percentage mortality. S. zeamais was more susceptible to the contact toxicity of the $P$. hispidinervum oil than $P$. aduncum oil with $\mathrm{LC}_{50}$ values of 0.51 and $2.87 \mathrm{~mL} \mathrm{~cm}^{-2}$ of the oil, respectively. The mortality rate was nearly $100 \%$ at $P$. hispidinervum oil concentrations of 20 and $30 \%$. With respect to the fumigant action, the weevil was more susceptible to the $P$. aduncum oil than to P. hispidinervum. A significant difference between oils mortality was observed only at 0.1 and $1.0 \%$ concentrations. For topical effect, similar values of the $\mathrm{LD}_{50}$ were obtained for essential oils. However, the mortality was higher with $P$. aduncum. Essential oils of the $P$. aduncum and $P$. hispidinervum possess insecticidal effect from $S$. zeamais, but the responses varied according to concentration of the oils and exposure methods.

Index terms: Piperaceae, botanical pesticides, dillapiol, safrole, natural insecticides.
\end{abstract}

\section{Introdução}

Entre as pragas que infestam grãos armazenados no Brasil, o gorgulho (Sitophilus zeamais Mots., 1855), (Coleoptera: Curculionidae) destaca-se como uma das mais prejudiciais, pelo grande número de hospedeiros, elevado potencial biótico, capacidade de penetração na massa de grãos e infestação cruzada, o que ocasiona danos, principalmente, aos grãos de milho, arroz e trigo (Gallo et al., 2002).
O controle desses insetos tem sido realizado em larga escala por meio de produtos químicos. Pesquisas atuais e o conhecimento dos efeitos indesejáveis do uso indiscriminado desses produtos, associado à preocupação dos consumidores quanto à qualidade de alimentos, têm incentivado estudos sobre novas técnicas de controle (Tavares, 2002).

Plantas com ação inseticida têm sido utilizadas como método alternativo de controle por meio de produtos com formulação em pó, óleos e extratos contra as principais 
pragas que ocorrem em produtos armazenados. Rodríguez (1999) relata o uso de folhas, sementes e do óleo de sementes de Azadirachta indica A. Juss. no controle de Rhyzopertha dominica (Fabr., 1792), Sitophilus oryzae (L.,1763), Tribolium castaneum Herbest., 1797, T. confusum, Callosobruchus chinenses L., C. maculatus e Oryzaephilus surinamensis (L., 1758), utilizando o pó a $1 \%$ de concentração, e o óleo, entre 0,2 e $0,5 \%$. Lale \& Abdulrahman (1999) também observaram que o óleo de sementes de nim reduz a oviposição e a emergência de adultos de C. maculatus. Maredia et al. (1992) verificaram alterações na sobrevivência de adultos de S. zeamais expostos ao pó e ao óleo de sementes de nim. O efeito inseticida do óleo e do pó de sementes de Piper guineense Schumach. \& Thonn., Dennettia tripetala (G. Baxer) e Aframomum melegueta (Roskoe) K. Schum, em relação a $S$. zeamais, foi verificado por Lale (1992). Esse autor observou que o óleo de $P$. guineense foi o mais tóxico, com $\mathrm{CL}_{50}$ de $2,63 \%$. Quanto ao pó, a atividade inseticida foi decrescente de $D$. tripetala, para A. meiegueta e P. guineense.

A ecologia química, ramo da ciência em crescimento, no qual as relações planta inseto e planta planta são examinadas em termos do efeito de substâncias sobre as funções biológicas, estabelece que essas substâncias são freqüentemente metabólitos secundários, que constituem verdadeiros sinais químicos nestas interações. Entre esses metabólitos, encontram-se os terpenos, especialmente os monoterpenos e seus análogos, que são componentes abundantes de óleos essenciais de muitas plantas superiores (Prates \& Santos, 2002). Esses óleos são compostos lipofilicos, com alto potencial para interferências tóxicas que protegem as plantas de ataques de insetos e infestação de parasitas (Simpson, 1995). Singh \& Upadhyay (1993) afirmaram que os óleos essenciais são fontes potenciais de inseticidas botânicos.

O objetivo deste trabalho foi avaliar a toxicidade de óleos essenciais de folhas de Piper hispidinervum C. DC e Piper aduncum L., em adultos de Sitophilus zeamais Motsch. (Coleoptera: Curculionidae).

\section{Material e Métodos}

Os experimentos foram conduzidos no Laboratório de Entomologia da Embrapa Acre, utilizando-se adultos de $S$. zeamais obtidos de uma criação massal em laboratório. As plantas de P. hispidinervum e P. aduncum foram coletadas de um banco de germoplasma da instituição.

\section{Obtenção e composição dos óleos essenciais}

Plantas adultas de $P$. aduncum e P. hispidinervum foram cortadas a $0,4 \mathrm{~m}$ do solo, separando-se, para processamento, as folhas e os ramos secundários. A massa vegetal foi submetida à secagem por seis dias em secador solar, até atingir 30\% de umidade, sendo revolvida duas vezes ao dia para facilitar a aeração (Pimentel et al., 1998). Posteriormente, o material foi submetido à extração por arraste forçado de vapor de água, utilizando-se o sistema de caldeira aquecida por gás, acoplada a um extrator (Simões \& Spitzer, 1999). Depois da condensação, a mistura de cada óleo essencial foi recebida em coletores comunicantes e separada por decantação. O rendimento dos óleos foi em média 1,8\%. A análise por cromatografia dos óleos essenciais foi realizada em cromatógrafo gasoso HP5890, coluna capilar de sílica fundida (DB - 5,30 m x $20 \mathrm{~mm}$ ), tendo hélio como gás de arraste e programação de temperatura de 80 a $140^{\circ} \mathrm{C}$ $\left(4^{\circ} \mathrm{C} /\right.$ minuto $)$ e de 140 a $240^{\circ} \mathrm{C}\left(20^{\circ} \mathrm{C} /\right.$ minuto $)$. A composição do óleo de $P$. aduncum foi de $73,97 \%$ de dilapiol, 3,92\% de safrol e 2,84\% de sarisan como componentes majoritários; quanto a $P$. hispidinervum, a análise cromatográfica apontou o safrol como componente majoritário, com 94,72\%.

\section{Curva de concentração-mortalidade}

Foram realizados testes preliminares com adultos de S. zeamais, para obtenção de faixas de respostas, ou seja, intervalos de concentração dos óleos que ocasionaram mortalidade do inseto desde próximo de zero até próximo de $100 \%$. Cada faixa de resposta foi obtida a partir da solução estoque de $50 \mathrm{~mL}$ do princípio ativo que, posteriormente, foi submetida a diluições seqüenciais em acetona p.a. até que fosse alcançada a concentração de $0,1 \%$. Dentro dessa ampla faixa de concentração, foram obtidas faixas mais estreitas de respostas. Na avaliação do efeito de contato em superfície contaminada, aplicação tópica e fumigação, as concentrações do óleo de $P$. hispidinervum e $P$. aduncum, variaram de 30,0 a $1,0 \%, 30,0$ a $0,1 \%$ e 5,0 a $0,1 \%$, respectivamente. Dentro desses intervalos, seguindo método descrito por Finney (1971), foram estabelecidas entre cinco e sete concentrações, além de um controle (solvente), usadas nos bioensaios definitivos de curva de concentração mortalidade e determinação da concentração com probabilidade de causar $50 \%$ de mortalidade ( $\mathrm{CL}_{50}$ e $\mathrm{DL}_{50}$ ) para cada óleo. 


\section{Bioensaios definitivos}

Em todos os bioensaios definitivos, as placas/potes de plástico foram mantidas em câmara climatizada à temperatura de $25 \pm 1^{\circ} \mathrm{C}$, umidade relativa de $70 \pm 5 \%$ e fotófase de 12 horas, até o momento da avaliação da mortalidade dos insetos. O tempo de exposição aos óleos foi de 48 horas considerando-se a mortalidade cumulativa no período.

\section{Contato em superfície papel-filtro}

Bioensaios de concentração mortalidade foram realizados com adultos de $S$. zeamais, usando-se placa de Petri $(9,0 \times 1,5 \mathrm{~cm})$ e papéis-filtro impregnados com $0,5 \mathrm{~mL}$ das seguintes concentrações: 30,0, 20,0, 10,0, 7,5, 5,0, 2,5 e 1,0\% dos óleos de $P$. hispidinervum e $P$. aduncum, diluídos em acetona (solvente). Depois da evaporação total do solvente, os pápeis-filtro foram colocados nas placas de Petri, que em seguida foram infestadas com 20 adultos por placa.

\section{Fumigação}

Os bioensaios in vivo foram instalados utilizando-se $30 \mathrm{~g}$ de grãos de milho (cv. BRS Sol da Manhã), que, depois da pesagem e determinação da umidade, foram colocados em potes de plástico descartáveis com capacidade para $100 \mathrm{~mL}$, com tampa hermética. Nessa massa de grãos, foram aplicados $2 \mathrm{~mL}$ das concentrações de $5,0,4,0,3,0,2,0,1,0$ e $0,1 \%$. Depois do revolvimento para homogeneização, deixou-se secar até a completa volatilização do solvente, para, em seguida, confinar, em cada frasco, 20 indivíduos adultos não sexados de S. zeamais.

\section{Inseticida por aplicação tópica}

Os adultos de $S$. zeamais foram anestesiados por congelamento $\left(-16^{\circ} \mathrm{C}\right)$ durante dois minutos, tempo suficiente apenas para que fossem manipulados. Os ensaios consistiram na aplicação tópica de $0,5 \mu \mathrm{L}$ das concentrações de 30, 20, 10, 7,5, 5 e 2,5 dos óleos com auxílio de uma microsseringa. Posteriormente, foram formados grupos de dez indivíduos em cada placa.

\section{Análise dos dados}

O delineamento experimental utilizado foi o inteiramente casualizado com quatro repetições, sendo cada bioensaio repetido três vezes, realizando-se análise conjunta dos resultados.

Em todos os bioensaios, os dados de mortalidade de concentração resposta foram submetidos à análise de Probit, utilizando-se o SAS (SAS Institute, 1989). Depois de estimadas, as $\mathrm{CL}_{50}$ e $\mathrm{DL}_{50}$ foram usadas como concentrações discriminatórias na detecção da toxicidade em relação a $S$. zeamais.

Os valores de mortalidade foram corrigidos pela fórmula de Abbott (1987), mortalidade (\%), e submetidos ao estudo de regressão de acordo com as concentrações de cada um dos óleos avaliados. Os dados de mortalidade de cada tratamento (concentração) dos óleos, em diferentes vias de contaminação, foram comparados mediante superposição das barras do erro-padrão da média de cada tratamento.

\section{Resultados e Discussão}

As curvas de concentração mortalidade do óleo de $P$. hispidinervum foram as que apresentaram a maior inclinação para os três efeitos testados (Tabela 1), demonstrando que adultos de $S$. zeamais responderam de forma mais homogênea a esse óleo e mais heterogênea ao óleo de P. aduncum. Valores altos de inclinação da curva indicam que pequenas variações na dose do óleo de $P$. hispidinervum promovem grandes variações na mortalidade, resultando em resposta homogênea da população a este produto (Atkins et al., 1973).

Tabela 1. Dados de inclinação das curvas de concentração-mortalidade, $\mathrm{CL}_{50}$ e $\mathrm{CL}_{95}, \chi^{2}$ e probabilidade dos óleos de Piper hispidinervum e Piper aduncum com efeitos de fumigação, contato e aplicação tópica em adultos de Sitophilus zeamais ${ }^{(1)}$.

\begin{tabular}{|c|c|c|c|c|c|c|c|c|}
\hline Aplicação & Óleo & GL & $\mathrm{n}$ & Inclinação + EPM & $\mathrm{CL}_{50}(\mathrm{IC} 95 \%)$ & $\begin{array}{c}\mathrm{DL}_{50}(\mathrm{IC} 95 \%) \\
\left(\mu \mathrm{L} \mathrm{g}^{-1} \text { de inseto) }\right.\end{array}$ & $\chi^{2}$ & Probabilidade \\
\hline \multirow[t]{2}{*}{ Fumigação } & P. hispidinervum & 4 & 480 & $1,31 \pm 0,15$ & $1,32(0,53-1,81)\left(\mu \mathrm{Lg}^{-1}\right)$ & - & 5,64 & 0,0001 \\
\hline & P. aduncum & 4 & 480 & $0,40 \pm 0,04$ & $0,56(0,09-1,28)\left(\mu \mathrm{L} \mathrm{g}^{-1}\right)$ & - & 66,90 & 0,0001 \\
\hline \multirow[t]{2}{*}{ Contato } & P. hispidinervum & 5 & 480 & $0,76 \pm 0,02$ & $0,51(0,42-0,62)\left(\mu \mathrm{L} \mathrm{cm}^{-2}\right)$ & - & 32,40 & 0,0001 \\
\hline & P. aduncum & 5 & 480 & $0,25 \pm 0,04$ & $2,87(2,17-4,20)\left(\mu \mathrm{L} \mathrm{cm}^{-2}\right)$ & - & 8,67 & 0,1220 \\
\hline \multirow[t]{2}{*}{ Tópica } & P. hispidinervum & 5 & 240 & $1,69 \pm 0,11$ & - & $0,04(0,03-0,04)$ & 0,17 & 0,9100 \\
\hline & P. aduncum & 5 & 240 & $1,01 \pm 0,07$ & - & $0,03(0,02-0,03)$ & 8,91 & 0,0300 \\
\hline
\end{tabular}

${ }^{(1)}$ GL: graus de liberdade; n: número de insetos usados no teste; EPM: erro-padrão da média; CL: concentração letal; IC: intervalo de confiança; DL: dose letal; $\chi^{2}$ : qui-quadrado. 
Os valores de $\mathrm{CL}_{50}$, estimados para os óleos de $P$. hispidinervum e P. aduncum, no efeito de fumigação, foram de 1,32 e 0,56 mL de óleo por g de grãos respectivamente, demonstrando que $S$. zeamais foi mais suscetível ao óleo de P. aduncum que ao de $P$. hispidinervum por meio desta via de contaminação (Tabela 1). Quanto aos testes de impregnação de papel-filtro, ocorreu o contrário: o óleo de P. hispidinervum apresentou menor $\mathrm{CL}_{50}\left(0,51 \mu \mathrm{L} \mathrm{cm}^{-2}\right.$ de óleo $)$ do que o de P. aduncum ( $2,87 \mu \mathrm{L} \mathrm{cm}^{-2}$ de óleo). Tapondjou et al. (2005), ao avaliar óleos essenciais de Eucalyptus saligna Sm. e Cupressus semprevirens L., em condições experimentais semelhantes a deste trabalho, consideraram promissores os valores de $\mathrm{CL}_{50}$ para $S$. zeamais (0,36 e $0,84 \mu \mathrm{L} \mathrm{cm}{ }^{-2}$ de óleo, respectivamente), sendo esses valores muito próximos ao obtido para o óleo essencial de $P$. hispidinervum.

No método de contato por aplicação tópica, os índices de toxicidade dos óleos foram semelhantes, sendo obtida $\mathrm{DL}_{50}$ de $0,04 \mu \mathrm{L} \mathrm{mg}^{-1}$ de inseto para $P$. hispidinervum e $0,03 \mu \mathrm{L} \mathrm{mg}^{-1}$ para $P$. aduncum.

Essas tendências se confirmaram nos valores de mortalidade de $S$. zeamais, evidenciando maior eficiência do óleo de $P$. aduncum por fumigação, nas concentrações de 0,1 e 1,0\%, embora com valores de mortalidade abaixo de $40 \%$ (Figura 1). Nas concentrações entre 2 e 5\%, não houve diferença significativa entre os valores de mortalidade causada pelos óleos avaliados. Foram obtidos valores acima de 70\% de mortalidade nas maiores concentrações avaliadas (4 e 5\%).

Resultados semelhantes foram observados sob o efeito de contato tópico, quando os valores de mortalidade de S. zeamais foram significativamente superiores com a aplicação do óleo de P. aduncum, em todas as concentrações avaliadas (Figura 1). Neste caso, valores de mortalidade acima de $70 \%$ somente foram alcançados nas concentrações acima de 10 e $20 \%$ para os óleos de $P$. hispidinervum e P. aduncum, respectivamente.

Não houve diferença significativa entre os valores de mortalidade de S. zeamais, quando os óleos essenciais foram avaliados por via de intoxicação de contato (papel-filtro), nas concentrações abaixo de 5\%, permanecendo a mortalidade abaixo de 40\% (Figura 1). As demais concentrações apresentaram diferenças nos valores de mortalidade causada pelo óleo de $P$. hispidinervum em relação ao de P. aduncum, variando de 90 a $100 \%$ entre as concentrações de 20 e 30\%, respectivamente. Tais concentrações podem ser consi- deradas elevadas, porém, para atingir esses valores de mortalidade em adultos de $S$. zeamais por contato (papel-filtro), Prates \& Santos (2002) utilizaram concentrações acima de $80 \%$ do óleo essencial de
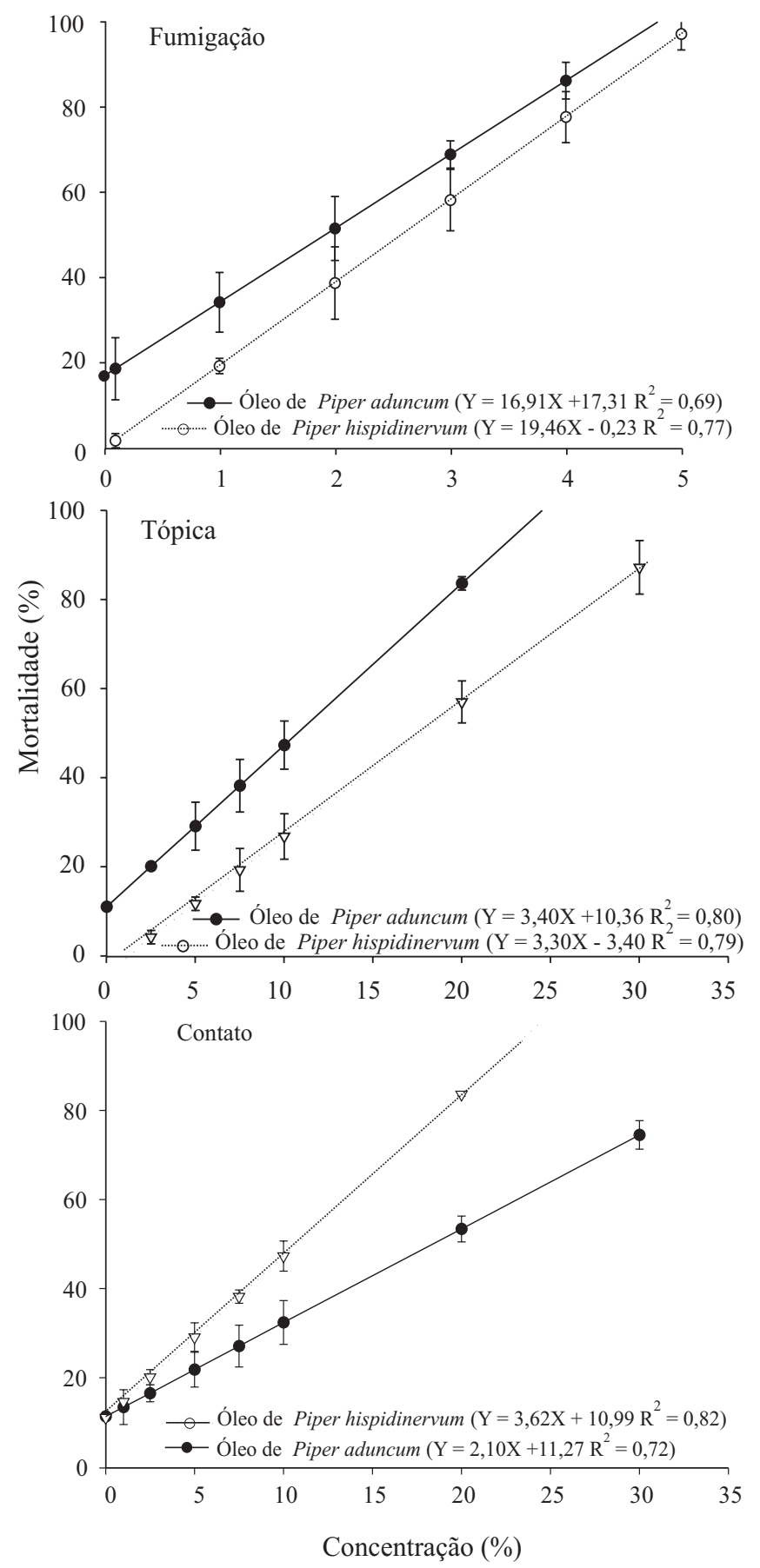

Figura 1. Mortalidade de adultos de Sitophilus zeamais em função das concentrações dos óleos de Piper aduncum e Piper hispidinervum aplicados via fumigação, aplicação tópica e por contato. Para todos os valores de $\mathrm{R}^{2}$, o nível de significância foi $\mathrm{p}<0,0001 \%$. 
Eucalyptus camaldulensis Denh. Em avaliações complementares, que incluíram a ingestão, além do efeito de contato, esses autores observaram mortalidade entre 90 e $100 \%$ em concentrações a partir de $20 \%$, o que evidencia a eficácia do óleo essencial de $P$. hispidinervum via contato (papel-filtro).

Estudos realizados por Bernard et al. (1995), com a espécie de $P$. aduncum, indicaram que essa espécie possui grande quantidade de ligninas, incluindo-se o dilapiol, e que, ao contrário da maioria das espécies desse gênero, não existem amidas relatadas como componentes dessa piperácea. A associação de ligninas ao grupo metilenedioxidofenil é uma característica das piperáceas, que se apresentam em grande número nas plantas e são consideradas importantes inibidores de monooxigenases dependentes do citocromo P450, utilizados, portanto, como sinergistas de inseticidas naturais (Mukerjee et al., 1979; Bernard et al., 1990). Para Regnault-Roger (1997), o efeito tóxico dos óleos envolve muitos fatores, entre os quais o ponto de entrada das toxinas, uma vez que os óleos podem ser inalados, ingeridos ou ainda absorvidos pelo tegumento dos insetos, podendo apresentar efeitos de contato, fumigação e fagoinibidor.

A bioatividade de safrol e isossafrol, compostos presentes em óleos essenciais de espécies vegetais, foi estudada também por Huang et al. (1999), em relação a Tribolium castaneum e S. zeamais. Os autores constataram que os insetos testados foram igualmente susceptíveis à ação de contato e fumigação no uso de safrol, e que adultos de $S$. zeamais foram mais tolerantes ao efeito fumigante de isossafrol em comparação a T. castaneum. Esses resultados foram confirmados quando a via de intoxicação foi por fumigação, uma vez que a concentração de 5\% causou praticamente a mortalidade de $100 \%$ dos adultos de S. zeamais, ao passo que, para atingir-se essa porcentagem de mortalidade por meio de aplicação tópica e por contato (papel-filtro), foi necessária concentração acima de $20 \%$.

\section{Conclusões}

1. Os óleos essenciais de Piper hispidinervum e $P$. aduncum apresentam efeito inseticida em Sitophilus zeamais e sua eficácia é dependente da via de intoxicação e da concentração do óleo aplicado.

2. P. hispidinervum é mais eficaz que $P$. aduncum pela via de intoxicação por contato em superfície contaminada.
3. $P$. aduncum é mais eficaz que $P$. hispidinervum pela via de intoxicação por fumigação e contato por aplicação tópica.

\section{Agradecimentos}

Ao CNPq, pelo financiamento das ações de pesquisa e pelas bolsas concedidas a Joelma Lima Vidal Estrela, Márcio Rodrigues Alécio e Marilene Santos de Lima.

\section{Referências}

ABBOTT, W.S. A method of computing the effectiveness of an insecticide. Journal of the American Mosquito Control Association, v.3, p.302-303, 1987.

ATKINS, E.L.; GREYWOOD, E.A.; MACDONALD, R.L. Toxicity of pesticides and other agricultural chemicals to honey bees: laboratory studies. Davis: University of California, 1973. 36p. (Technical bulletin, M-16).

BERNARD, C.B.; ARNASON, J.T.; PHILOGÈNE, B.J.R.; LAM, J.; WADDELL, T. In vivo effect of mixtures of allelochemicals on the life cycle of the European corn borer, Ostrinia nubilalis. Entomologia Experimentalis et Applicata, v.57, p.17-22, 1990.

BERNARD, C.B.; KRISHANMURTY, H.G.; CHAURET, D.; DURST, T.; PHILOGÈNE, B.J.R.; SÁNCHEZ-VINDAS, P.; HASBUN, C.; POVEDA, L.; SAN ROMÁN, L.; ARNASON, J.T. Insecticidal defenses of Piperaceae from the neotropics. Journal of Chemical Ecology, v.21, p.801-814, 1995.

FINNEY, D.J. Probit analysis. $3^{\text {rd }}$ ed. London: Cambridge University Press, 1971. 333p.

GALLO, D.; NAKANO, O.; SILVEIRA NETO, S.; CARVALHO, R.P.L.; BATISTA, G.C. de; BERTI FILHO, E.; PARRA, J.R.P.; ZUCCHI, R.A.; ALVES, S.B.;VENDRAMIM, J.D.; MARCHINI, L.C.; LOPES, J.R.S.; OMOTO, C. Entomologia agrícola. Piracicaba: Fealq, 2002. 920p.

HUANG, Y.; HO, S.H.; KINI, R.M. Bioactivities of safrole and isosafrole on Sitophilus zeamais (Coleoptera: Curculionidae) and Tribolium castaneum (Coleoptera: Tenebrionidae). Journal of Economic Entomology, v.92, p.676-683, 1999.

LALE, N.E.S. A laboratory study of the comparative toxicity of products from three spices to the maize weevil. Postharvest Biology and Technology, v.2, p.61-64, 1992.

LALE, N.E.S.; ABDULRAHMAN, H.T. Evaluation of neem (Azadirachta indica A. Juss.) seed oil obtained by different methods and neem powder for the management of Callosobruchus maculatus (F.) (Coleoptera: Bruchidae) in stored cowpea. Journal of Stored Products Research, v.35, p.135-143, 1999.

MAREDIA, K.M.; SEGURA, O.L.; MIHM, J.A. Effects of neem, Azadirachta indica, on six species of maize insect pests. Tropical Pest Management, v.38, p.190-195, 1992.

MUKERJEE, S.K.; SAXENA, V.S.; TOMAR, S.S. New methylenedioxyphenyl synergists for pyrethrins. Journal of Agricultural and Food Chemistry, v.27, p.1209-1211, 1979. 
PIMENTEL, F.A.; CABRAL, W.G.; SILVA, M.R. da; PINHEIRO, P.S.N. Processo de secagem de biomassa de pimenta longa (Piper hispidinervum). Rio Branco: Embrapa-CPAF, 1998. 2p. (EmbrapaCPAF. Comunicado técnico, 98).

PRATES, H.T.; SANTOS, J.P. dos. Óleos essenciais no controle de pragas de grãos armazenados. In: LORINI, I.; MIIKE, L.H.; SCUSSEL, V.M. (Org.). Armazenagem de grãos. Campinas: IBG, 2002. p.443-461.

REGNAULT-ROGER, C. The potential of botanical essential oils for insect pest control. Integrated Pest Management Reviews, v.2, p.25-34, 1997.

RODRÍGUEZ, C. Receitas de nim Azadirachta indica (Meliaceae) contra plagas. In: SIMPOSIO NACIONAL SOBRE SUBSTANCIAS VEGETALES Y MINERALES EN EL COMBATE DE PLAGAS, 5., 1999, Aguascalientes. Memorias. Aguascalientes: SME, 1999. p.39-59.

SAS INSTITUTE. (Cary, Estados Unidos). SAS user's guide: statistics. Version 6.12. Cary, NC, 1998. 943p.

SIMÕES, C.M. de O.; SPITZER, V. Óleos voláteis. In: SIMÕES, C.M. de O.; SCHENKEL, E.P.; GOSMANN, G.; MELLO, J.C.P. de; MENTZ, L.A.; PETROVICK, P.R. (Org.). Farmacognosia: da planta ao medicamento. Florianópolis: UFSC, 1999. p.387-416.

SIMPSON, B.B. Spices, herbs and perfumes. In: SIMPSON, B.B.; OGORZALY, M.C. (Ed.). Economic botany: plants in our world. $2^{\text {nd }}$ ed. New York: McGraw-Hill, 1995. p.278-301.

SINGH, G.; UPADHYAY, R.K. Essential oils: a potent source of natural pesticides. Journal of Scientific and Industrial Research, v.52, p.676-683, 1993.

TAPONDJOU, A.L.; ADLER, C.; FONTEM, D.A.; BOUDA, H.; REICHMUTH, C. Bioactivities of cymol and essential oils of Cupressus sempervirens and Eucalyptus saligna against Sitophilus zeamais Moutschulsky and Tribolium confusum du Val. Journal of Stored Products Research, v.41, p.91-102, 2005.

TAVARES, M.A.G.C. Bioatividade da erva-de-santa-maria, Chenopodium ambrosoides $\mathbf{L}$. (Chenopodiaceae), em relação a Sitophilus zeamais Mots., 1855 (Col: Curculionidae). 2002. 59p. Dissertação (Mestrado) - Escola Superior de Agricultura Luiz de Queiroz, Piracicaba.

Recebido em 29 de novembro de 2004 e aprovado em 28 de setembro de 2005 